\title{
Hospital-acquired pneumonia: microbiological data and potential adequacy of antimicrobial regimens
}

\author{
O. Leroy*, P. Giradie*, Y. Yazdanpanah*, H. Georges*, S. Alfandari*, V. Sanders*, P. Devos", \\ G. Beaucaire*
}

Hospital-acquired pneumonia: microbiological data and potential adequacy of antimicrobial regimens. O. Leroy, $P$. Giradie, Y. Yazdanpanah, $H$. Georges, $S$. Alfandari, V. Sanders, P. Devos, G. Beaucaire. C ERS Journals Ltd 2002.

ABSTRACT: Adequate antimicrobial therapy is a main approach employed to decrease the mortality associated with hospital-acquired pneumonia (HAP). All methods that optimise empirical treatment without increasing antibiotic selective pressure are relevant. Categorisation of patients according to HAP time of onset, severity and risk factors (American Thoracic Society (ATS) classification) or duration of mechanical ventilation and prior antibiotics (Trouillet's classification) are two such methods. The aim of this study was to catagorise patients with HAP according to these classifications and to determine the frequency of resistant pathogens and the most adequate antimicrobial regimens in each group.

A total 124 patients with bacteriologically proven HAP were studied. The ATS classification categorised patients by increasing frequency of resistant pathogens from $0-30.3 \%$. The ATS empirical antibiotic recommendations appeared valid but proposed combinations including vancomycin for $\mathbf{7 2 . 5 \%}$ of patients. Trouillet's classification categorised patients into four groups with a frequency of resistant pathogens from 4.9-35.6\%. Vanomycin was proposed for $48.5 \%$ of patients.

The American Thoracic Society classification appears to be more specific than Trouillet's for predicting the absence of resistant causative pathogens in hospitalacquired pneumonia but could lead to a greater use of vanomycin. Stratification combining the two classifications is an interesting alternative. Eur Respir J 2002; 20: 432-439.
*Intensive Care Unit and Infectious Diseases Dept, Lille University Medical School, Chatiliez Hospital, Tourcoing, and ${ }^{\#}$ Dept of Biostatistics, Lille University Medical School, Lille, France.

Correspondence: O. Leroy, Service de Réanimation Médicale et Maladies Infectieuses, Centre Hospitalier, Rue du President Coty, 59208, Tourcoing, France.

Fax: 33320694439

E-mail: oyleroy@ch-tourcoing.fr

Keywords: Antibiotics

intensive care

nosocomial pneumonia

pneumonia

resistance

Received: August 232001

Accepted after revision: April 112002
Hospital-acquired pneumonia (HAP) remains the most severe nosocomial infection in intensive care units (ICUs). Although mortality rates vary from one study to another and its prognostic impact is debated [1-7], it is recognised that one-third to one-half of all HAP deaths are directly attributable to infection [8]. Some factors influencing mortality have been identified. Bacteraemia and Pseudomonas aeruginosa or Acinetobacter spp. as causative agents increase mortality [3, 9]. Conversely, adequate and prompt initial antimicrobial therapy reduces mortality $[10,11]$.

Treatment instituted before knowing the aetiology and antimicrobial sensitivities is empirical. In order to choose the correct antibiotics, different means could be proposed. Firstly, guidelines such as those proposed by the American Thoracic Society (ATS) [8] can be used. These guidelines are based on patient classification into three categories according to the severity of HAP, the time of onset and the presence or absence of specific risk factors. In each group, different possible causative pathogens are incriminated and different antimicrobial regimen are proposed. Secondly, specific epidemiological data from each ICU can be studied and hence antibiotic regimens can be tailored. Such work performed by TROUILLET et al. [12], who studied patients with ventilator-associated pneumonia (VAP). According to the duration of mechanical ventilation (MV) prior to the VAP onset and the presence or absence of prior antibiotic(s), patients were separated into four different groups in which the frequency of potentially resistant organisms and their antimicrobial susceptibility varied.

In addition to the adequacy of the initial antimicrobial therapy, physicians must consider the problem of bacterial resistance. In particular, the use of widespectrum agents and vancomycin have been incriminated in the occurrence and increase of resistance [13].

Consequently, all methods optimising the choice of an adequate HAP antimicrobial treatment and decreasing the selective pressure appear relevant.

The aim of this study was to retrospectively categorise all patients exhibiting HAP, in the current authors unit, according to the ATS [8] and TrouILlet et al. [12] classifications and to determine microbial epidemiology and potentially the most adequate antimicrobial regimen.

\section{Methods and materials}

\section{Selection of patients}

From January 1994-December 1999, all patients admitted to the current authors ICU for HAP or exhibiting HAP during their ICU stay were enrolled. 
HAP was considered when new and/or progressive chest radiographical infiltrates occurred $\geqslant 48 \mathrm{~h}$ after hospital admission, in conjunction with at least two of the following criteria: purulent respiratory secretions, temperature $>38.5$ or $<35^{\circ} \mathrm{C}$, blood leucocyte count $>10,000$ or $<1,500 \cdot \mathrm{mm}^{-3}$. Only patients with bacteriologically documented HAP were studied. Establishing an aetiological diagnosis required isolation of bacteria in significant quantity from a sample of lower respiratory tract secretions (endotracheal aspiration $>1 \times 10^{6}$ colony forming units $(\mathrm{cfu}) \cdot \mathrm{mL}^{-1}$, protected brush catheter $>1 \times 10^{3} \mathrm{cfu} \cdot \mathrm{mL}^{-1}$ or bronchoalveolar lavage $>1 \times 10^{4} \mathrm{cfu} \cdot \mathrm{mL}^{-1}$ ) or isolation of a definitive pathogen from a blood or pleural fluid culture. These latter cultures were considered significant when the same organism, as recovered from the sample of respiratory secretion, was identified.

On ICU admission, age and sex as well as severity of illness and vital sign abnormalities were recorded, and then evaluated by Simplified Acute Physiology Score (SAPS II) [14]. When HAP occurred, the time of onset from hospital admission, temperature, chest radiographical involvement and leucocyte count were recorded.

\section{Definition of groups of patients}

The ATS guidelines [8] stratify patients with HAP into three groups, according to its severity, time of onset, and presence or absence of specific risk factors. All patients exhibiting HAP, whether the pneumonia is ventilator associated or not, are classified. Group 1 includes "patients without unusual risk factors who present with mild-to-moderate HAP with onset at any time during hospitalisation or severe HAP with early onset". Group 2 includes "patients with specific risk factors who present with mild-to-moderate HAP occurring at any time during hospitalisation". Group 3 includes "patients with severe HAP either of early onset with specific risk factors or of late onset" [8]. As previous antimicrobial treatment is a risk factor for selecting resistant pathogens, subjects in group 3 were further divided into subgroups according to the absence or presence of prior antibiotic(s) within 1 month before HAP.

TROUILLET et al. [12] proposed a classification for patients with VAP based on duration of MV $(<7$ days or $\geqslant 7$ days before VAP onset) and presence or absence of antibiotic treatment within the 15 days preceding VAP. Four groups were defined. In the present study, any antibiotic treatment within 1 month before HAP onset was taken into account and all patients with HAP were studied, whether ventilator associated or not. Consequently, nonventilated and ventilated patients with HAP occurring before the seventh day of MV were included in groups A and B. In group A, patients had not received antibiotic(s) within 1 month preceding HAP, while the subjects in group B had. Groups C and D included ventilated patients with $\mathrm{MV}$ duration $\geqslant 7$ days before HAP onset. In group $C$, patients had not received antibiotic(s) within the month preceding HAP while in group D they had.

\section{Microbial epidemiology}

In each HAP episode, all significant isolates were identified by standard laboratory techniques. For each pathogen, its antimicrobial susceptibility was studied. Criteria propsed by the Comité de l'Antibioprogramme de la Société Française de Microbiologie [15] were used. Antibiotics tested were amoxycillin/clavulanic acid, cefotaxime, ceftazidime, cefepime, piperacillin, piperacillin/tazobactam, imipenem, ciprofloxacin, vancomycin and amikacin.

According to the definition of Trouillet et al. [12], methicillin-resistant Staphylococcus aureus, P. aeruginosa, Acinetobacter spp. and Stenotrophomonas maltophilia were considered as "potentially resistant" bacteria. For the purpose of this study, methicillinresistant $S$. aureus, ticarcillin-resistant $P$. aeruginosa, extended-spectrum $\beta$-lactamase producing Enterobacteriaceae and all S. maltophilia and Acinetobacter spp. strains were considered as "truly resistant" bacteria.

The distribution of causative organisms, "potentially resistant" and "truly resistant" bacteria, was studied in each group defined by the ATS [8] and TrouiLlet et al. [12] classifications.

\section{Potential adequacy of antibiotic regimens}

An adequate antimicrobial regimen for HAP was defined as the use of at least one antibiotic to which all isolates were susceptible in vitro. In the presence of $P$. aeruginosa, a combination of at least two active agents was required [16]. In the presence of methicillinresistant $S$. aureus, vancomycin was required.

To assess the potential adequacy of antimicrobial regimens, an analysis based on three steps was performed. First, the susceptibility of all organisms was determined. Second, HAP episodes and antibiotic monotherapy were considered. Betalactams were always considered inadequate when $P$. aeruginosa and/or methicillin-resistant $S$. aureus were implicated as pathogen(s) or copathogen(s). For other organisms, any antibiotic was inadequate if the pathogen or one of the pathogens was resistant to this antibiotic. The third step was to study antimicrobial combinations in a HAP episode. All pathogens implicated in an episode and the respective adequacy of each antibiotic used in combination were examined. For all pathogens, except $P$. aeruginosa and methicillin-resistant $S$. aureus, a regimen was adequate when no organism was resistant to all antibiotics used in combination. In the presence of $P$. aeruginosa, two combined active agents were required. In the presence of methicillinresistant $S$. aureus, vancomycin was required.

Among regimens recommended by the ATS [8], some antibiotics were not available in the current author's hospital. Consequently, only the adequacy of amoxycillin/clavulanic acid, piperacillin/tazobactam, cefotaxime and ciprofloxacin for patients classified in ATS group 1 were tested. In group 3, piperacillin, piperacillin/tazobactam, ceftazidime, cefepime and imipenem, combined with amikacin or ciprofloxacin, and each combination with and without vancomycin were tested. 
In the groups determined by the classification by Trouillet et al. [12], the adequacy of amoxycillin/ clavulanic acid, cefotaxime, piperacillin, piperacillin/ tazobactam, ceftazidime, cefepime or imipenem, used as single agent, were tested. Then, all of them combined with amikacin or ciprofloxacin were tested. Finally, all these combinations with vancomycin were tested.

The results were expressed as percentages of adequate regimens in HAP episodes.

\section{Statistical analysis}

The distribution of organisms was compared in groups according to the classification used. Either the Chi-squared test or the Fisher's exact test was used. A p-value $\leqslant 0.05$ was considered as a significant difference.

\section{Results}

\section{Study population}

During the study period, 172 HAP episodes were evaluated. Pathogen(s) were identified in 124 episodes that occurred in 124 patients (mean age $64 \pm 14$ yrs, 89 males). On ICU admission, the mean SAPS II was $44 \pm 12$. The mean time of HAP onset from hospital admission was $15.4 \pm 12.2$ days. A total 103 of the HAP cases $(83 \%)$ were VAP. The mean duration of MV before HAP onset was $10.3 \pm 11.0$ days. When HAP occurred, the temperature was $>38.5^{\circ} \mathrm{C}$ in 107 patients and $<35^{\circ} \mathrm{C}$ in four. All patients had purulent respiratory secretions. The leucocyte count was $>10,000 \mathrm{~mm}^{-3}$ in 109 cases and $<1,500 \mathrm{~mm}^{-3}$ in one case. Radiographical infiltrates were bilateral in 39 patients.
During the HAP episode, MV was required for 117 patients.

\section{Distribution of patients}

According to the ATS classification [8], no patient was included in group 2. Six patients were included in group 1 and 118 in group 3. In group 3, 90 patients had received antibiotic(s) within 1 month prior to HAP onset. In the classification by TROUILLET et al. [12], 31, 33, 3 and 57 patients were included in groups $\mathrm{A}, \mathrm{B}, \mathrm{C}$ and $\mathrm{D}$, respectively. In groups $\mathrm{A}$ and $\mathrm{B}$ respectively, 7 of $31(22.6 \%)$ and 13 of $33(39.4 \%)$ patients were nonventilated when HAP occurred.

When patients were analysed for both the ATS [8] and Trouillet et al. [12] classifications, it was observed that all patients $(n=6)$ included in group 1 were included in group A. In group $3(n=118)$, the distribution of patients in groups $\mathrm{A}, \mathrm{B}, \mathrm{C}$ and $\mathrm{D}$ was $25,33,3$ and 57, respectively.

Microbiological data and distribution of microorganisms responsible for hospital-acquired pneumonia

A total 154 pathogens were isolated (table 1). Infection was polymicrobial in 32 cases. The main organisms were $P$. aeruginosa $(31.2 \%)$, Enterobacteriaceae spp. (20.8\%), S. aureus $(18.8 \%)$ with $33 \%$ methicillin-resistant strain, Haemophilus influenzae (6.5\%), Streptococcus pneumoniae (5.8\%), Acinetobacter spp. $(5.8 \%)$ and $S$. maltophilia $(5.2 \%)$.

The distribution of causative pathogens, according to the ATS classification [8], is detailed in table 1 . In group 3, almost one-half of organisms were "potentially resistant", but only $23.4 \%$ were "truly

Table 1.-Bacteria isolated in the 124 episodes of hospital-acquired pneumonia

\begin{tabular}{|c|c|c|c|c|c|c|c|}
\hline & Total & Group 1 & Group 3 & Group A & Group B & Group C & Group D \\
\hline Number of episodes & 124 & 6 & 118 & 31 & 33 & 3 & 57 \\
\hline Number of bacteria & 154 & 8 & 146 & 41 & 36 & 4 & 73 \\
\hline \multicolumn{8}{|l|}{ Organisms } \\
\hline S. pneumoniae & 9 & 2 & 7 & 5 & 2 & & 2 \\
\hline Streptococcus spp. & 2 & & 2 & 1 & & & 1 \\
\hline MSSA & 19 & 3 & 16 & $12^{\bullet}$ & 1 & 1 & 5 \\
\hline MRSA & 10 & & 10 & & 1 & 1 & $8^{+}$ \\
\hline $\mathrm{CNS}$ & 1 & & 1 & & 1 & & \\
\hline M. catarrhalis & 5 & 1 & 4 & 4 & & & 1 \\
\hline E. coli & 8 & & 8 & 4 & 3 & & 1 \\
\hline Enterobacter spp. & 7 & & 7 & 1 & 4 & & 2 \\
\hline Klebsiella spp. & 5 & & 5 & & 1 & & 4 \\
\hline Serratia spp. & 7 & & 7 & & 2 & 1 & 4 \\
\hline Proteus spp. & 5 & & 5 & & 1 & & 4 \\
\hline H. influenzae & 10 & 2 & 8 & 8 & 1 & & 1 \\
\hline P. aeruginosa & 48 & & 48 & 5 & $16^{\S, f}$ & 1 & $26^{f}$ \\
\hline S. maltophilia & 8 & & 8 & 1 & 3 & & 4 \\
\hline Acinetobacter spp. & 9 & & 9 & & & & 9 \\
\hline Corynebacterium spp. & 1 & & 1 & & & & 1 \\
\hline
\end{tabular}

S. pneumoniae: Streptococcus pneumoniae; MSSA: methicillin sensitive Staphylococcus aureus; MRSA: methicillin resistant Staphylococcus aureus; CNS: coagulase negative staphylococci; M. catarrhalis: Moraxcella catarrhalis; E. coli: Escherichia coli; H. influenzae: Haemophilus influenzae; P. aeruginosa: Pseudomonas aeruginosa; S. maltophilia: Stenotrophomonas maltophilia. ${ }^{\#}: \mathrm{p}<0.05$ groups $\mathrm{A}+\mathrm{B}$ versus groups $\mathrm{C}+\mathrm{D} ;{ }^{\uparrow}: \mathrm{p}<0.01$ versus group $\mathrm{B}$ and $\mathrm{p}<0.01$ versus group $\mathrm{D} ;{ }^{+}: \mathrm{p}<0.001$ versus group $\mathrm{A}$ and and versus group $\mathrm{B} ;{ }^{\S}: \mathrm{p}<0.01$ versus group $\mathrm{A} ;{ }^{f}: \mathrm{p}<0.01$ groups $\mathrm{B}+\mathrm{D}$ versus groups $\mathrm{A}+\mathrm{C}$. 
Table 2. - Numbers and percentages of "potentially" and "truly resistant" bacteria isolated in 124 episodes of hospitalacquired pneumonia according to the American Thoracic Society classification

\begin{tabular}{|c|c|c|c|c|c|}
\hline & \multirow[t]{2}{*}{ Total } & \multirow[t]{2}{*}{ Group 1} & \multicolumn{3}{|c|}{ Group 3} \\
\hline & & & Overall & No prior antibiotics & Prior antibiotics \\
\hline Organisms $\mathrm{n}$ & 154 & 8 & 146 & 37 & 109 \\
\hline "Potentially resistant" bacteria & $75(48.8)$ & 0 & $75(48.8)$ & $8(21.6)^{\#}$ & $67(61.5)$ \\
\hline P. aeruginosa & $48(31.2)$ & 0 & $48(31.2)$ & $6(16.2)$ & $42(38.5)$ \\
\hline Acinetobacter spp. & $9(5.8)$ & 0 & $9(5.8)$ & 0 & $9(8.3)$ \\
\hline S. maltophilia & $8(5.2)$ & 0 & $8(5.2)$ & $1(2.7)$ & 7 (6.4) \\
\hline MRSA & $10(6.5)$ & 0 & $10(6.5)$ & $1(2.7)$ & $9(8.3)$ \\
\hline "Truly resistant" bacteria & $36(23.4)$ & 0 & $36(23.4)$ & $3(8.1)^{9}$ & $33(30.3)$ \\
\hline Ticarcillin resistant $P$. aeruginosa & $5(3.2)$ & 0 & $5(3.2)$ & $1(2.7)$ & $4(3.7)$ \\
\hline Acinetobacter spp. & $9(5.8)$ & 0 & $9(5.8)$ & 0 & $9(8.3)$ \\
\hline S. maltophilia & $8(5.2)$ & 0 & $8(5.2)$ & $1(2.7)$ & $7(6.3)$ \\
\hline MRSA & $10(6.5)$ & 0 & $10(6.5)$ & $1(2.7)$ & $9(8.3)$ \\
\hline ESBL producing enterobacteriaceae & $4(2.6)$ & 0 & $4(2.6)$ & 0 & $4(3.7)$ \\
\hline
\end{tabular}

Data are presented as n (\%). P. aeruginosa: Pseudomonas aeruginosa; S. maltophilia: Stenotrophomonas maltophilia; MRSA: methicillin resistant Staphylococcus aureus; ESL: extended-spectrum $\beta$-lactamase. ${ }^{\#}$ : $\mathrm{p}<0.0001$ versus patients with prior antibiotics; ${ }^{\uparrow}: \mathrm{p}=0.003$ versus patients with prior antibiotics.

resistant" (table 2). If the presence or absence of prior antibiotic(s) were taken into account, the incidence of "potentially resistant" pathogens (21.6 versus $61.5 \%$, $\mathrm{p}<0.0001)$ and "truly resistant" pathogens $(8.1$ versus $30.3 \%, \mathrm{p}=0.003$ ) was significantly less in the subgroup of patients without prior antibiotics (table 2). Thus in the patients in this study, ATS classification [8] was able to detect HAP episodes due to resistant organisms with a negative predictive value of $100 \%$ since no "potentially or truly resistant" organism was implicated in group 1.

In the classification by Troullnet et al. [12], $S$. pneumoniae was more frequently isolated when the duration of MV was $<7$ days (groups $\mathrm{A}+\mathrm{B}$ versus $\mathrm{C}+\mathrm{D}, \mathrm{p}<0.05)$. Methicillin-susceptible $S$. aureus was found more often in group A than in B $(\mathrm{p}<0.01)$ and D $(\mathrm{p}<0.01)$. Methicillin-resistant $S$. aureus was predominant in group $\mathrm{D}(\mathrm{p}<0.001)$. $P$. aeruginosa was incriminated as a causative bacterium in all groups. In patients with a duration of MV of $<7$ days and without prior antibiotics (group A), $P$. aeruginosa accounted for $12.2 \%$ of the total number of bacteria.
However, $P$. aeruginosa was isolated more frequently when HAP occured after prior antibiotic(s) (groups $\mathrm{B}+\mathrm{D}$ versus $\mathrm{A}+\mathrm{C}, \mathrm{p}<0.01)$.

The distribution of "potentially and truly resistant" pathogens is reported in table 3 . In group A, the incidence of "potentially resistant" organisms was low $(14.7 \%)$. In groups B, C and D, the incidence of "potentially resistant" organisms was $>50 \%$. Finally, an increasing incidence of "truly resistant" pathogens from group A $(4.9 \%)$ to groups B $(19.5 \%)$, C $(25 \%)$ and $\mathrm{D}(35.6 \%)$ was found. Thus in this series, this classification was unable to distinguish a group without resistant causative organisms.

Potential adequacy of antimicrobial regimens in hospital-acquired pneumonia episodes

For patients in ATS group 1, adequacy levels of amoxycillin/clavulanic acid, piperacillin/tazobactam, cefotaxime and ciprofloxacin were 100, 100, 100 and $50 \%$, respectively. In group 3 , adequacy levels are

Table 3. - Numbers and percentages of "potentially" and "truly resistant" bacteria isolated in 124 episodes of hospitalacquired pneumonia classified according to the duration of mechanical ventilation and prior antimicrobial therapy

\begin{tabular}{|c|c|c|c|c|c|}
\hline & Total & Group A & Group B & Group C & Group D \\
\hline Organisms $\mathrm{n}$ & 154 & 41 & 36 & 4 & 73 \\
\hline "Potentially resistant" bacteria & $75(48.8)$ & $6(14.7)$ & $20(55.6)$ & $2(50)$ & $47(64.4)$ \\
\hline P. aeruginosa & $48(31.2)$ & $5(12.2)$ & $16(44.5)$ & $1(25)$ & $26(35.6)$ \\
\hline Acinetobacter spp. & $9(5.8)$ & 0 & 0 & 0 & $9(25)$ \\
\hline S. maltophilia & $8(5.2)$ & $1(2.4)$ & $3(8.3)$ & 0 & $4(11.1)$ \\
\hline MRSA & $10(6.5)$ & 0 & $1(2.8)$ & $1(25)$ & $8(22.2)$ \\
\hline "Truly resistant" bacteria & $36(23.4)$ & $2(4.9)$ & $7(19.5)$ & $1(25)$ & $26(35.6)$ \\
\hline Ticarcillin resistant $P$. aeruginosa & $5(3.2)$ & $1(2.4)$ & 0 & 0 & $4(5.5)$ \\
\hline Acinetobacter spp. & $9(5.8)$ & 0 & 0 & 0 & $9(12.3)$ \\
\hline S. maltophilia & $8(5.2)$ & $1(2.4)$ & $3(8.3)$ & 0 & $4(5.5)$ \\
\hline MRSA & $10(6.5)$ & 0 & $1(2.8)$ & $1(25)$ & $8(11)$ \\
\hline ESBL producing Enterobacteriaceae & $4(2.6)$ & 0 & $3(8.3)$ & 0 & $1(1.4)$ \\
\hline
\end{tabular}

Data are presented as n (\%). P. aeruginosa: Pseudomonas aeruginosa; S. maltophilia: Stenotrophomonas maltophilia; MRSA: methicillin resistant Staphylococcus aureus; ESL: extended-spectrum $\beta$-lactamase. 
Table 4.-Potential adequacy of selected antimicrobial combination therapies in 118 episodes of hospital-acquired pneumonia (group 3 according to American Thoracic Society classification)

\begin{tabular}{lcc}
\hline & $\begin{array}{c}\text { Patients without prior } \\
\text { antimicrobial therapy \% }\end{array}$ & $\begin{array}{c}\text { Patients with prior } \\
\text { antimicrobial therapy \% }\end{array}$ \\
\hline Episodes of HAP n & 28 & 90 \\
Combinations with amikacin & & 56.7 \\
Piperacillin & 78.6 & 63.3 \\
Piperacillin+vancomycin & 82.1 & 62.2 \\
Piperacillin/tazobactam & 82.1 & 68.9 \\
Piperacillin/tazobactam+vancomycin & 85.7 & 61.1 \\
Ceftazidime & 92.9 & 68.9 \\
Ceftazidime+vancomycin & 96.4 & 63.3 \\
Cefepime & 92.9 & 70.0 \\
Cefepime+vancomycin & 96.4 & 62.2 \\
Imipenem & 85.7 & 67.9 \\
Imipenem+vancomycin & 89.3 & 47.8 \\
Combinations with ciprofloxacin & & 55.6 \\
Piperacillin & 75.0 & 60.0 \\
Piperacillin+vancomycin & 75.0 & 67.8 \\
Piperacillin/tazobactam & 78.6 & 56.7 \\
Piperacillin/tazobactam+vancomycin & 78.6 & 65.6 \\
Ceftazidime & 89.3 & 61.1 \\
Ceftazidime+vancomycin & 92.9 & 68.9 \\
Cefepime & 85.7 & 62.2 \\
Imipenem & 89.3 & 68.9 \\
Imipenem+vancomycin & 85.7 & 89.3 \\
\hline
\end{tabular}

reported in table 4. In the subgroup of patients without prior antibiotic(s), only cefepime and ceftazidime combined with amikacin reached a level of $>90 \%$. With the addition of vancomycin, the improvement of adequacy level was always $<5 \%$. For patients with prior antibiotic(s), levels were lower, ranging from $47.8 \%$ for piperacillin/ciprofloxacin combination to $70 \%$ for cefepime/amikacin/vancomycin combination.

The potential adequacy of regimens in groups of Trouillet's et al. [12] classification is reported in table 5. In group A, all betalactams except piperacillin, used as monotherapy, reached a level of $>74 \%$. Levels of piperacillin/tazobactam, ceftazidime, cefepime and imipenem, combined with amikacin were $>90 \%$. Combinations with ciprofloxacin had lower levels than combinations with amikacin. The addition of vancomycin to betalactam/amikacin or ciprofloxacin combinations left levels unchanged. In group B, no betalactam used as monotherapy reached a level $>45 \%$. Levels reached by piperacillin, piperacillin/tazobactam, ceftazidime, cefepime or imipenem, combined with amikacin, were $>75 \%$. When betalactams were combined with ciprofloxacin, only imipenem had a level of $>75 \%$. Levels reached by betalactam/ciprofloxacin combinations were lower than respective levels reached by the same betalactam combined with amikacin. Finally, when vancomycin was added to betalactam/amikacin or ciprofloxacin combinations, improvement of levels was close to $3 \%$. In group $\mathrm{C}$, there were only three episodes, a figure too low to comment. In group $\mathrm{D}$, levels of all regimens were low, close to $30 \%$ for monotherapies, to $50 \%$ for the most adequate betalactam combined with amikacin, and to $55 \%$ for the most adequate betalactam combined with ciprofloxacin. When vancomycin was added, improvement of levels was near to $10 \%$. However, the best regimen exhibited a level of $<70 \%$.

\section{Discussion}

In this study, the classifications of the ATS [8] and Trouillet et al. [12] were used to categorise patients. With the ATS classification [8], patients were included into classes with an increasing frequency of "truly resistant" pathogens from $0-30.3 \%$. Current ATS therapeutic recommendations appeared valid, with a treatment based on a single antibiotic (amoxycillin/ clavulanic acid or piperacillin/tazobactam or cefotaxime) for $5 \%$ of patients, two antibiotics (broad spectrum betalactam combined with amikacin or ciprofloxacin) for $22.5 \%$ of patients and three antibiotics (broad spectrum betalactam plus amikacin or ciprofloxacin plus vancomycin) for $72.5 \%$ of patients. With the classification of TROUILLET et al. [12], patients were categorised into four groups with an increasing frequency of "truly resistant" pathogens from 4.9-35.6\%. Using this classification, an empirical antimicrobial treatment, based on two antibiotics (broad spectrum betalactam combined with amikacin), could be proposed for $51.5 \%$ of patients. Combinations, including a broad-spectrum betalactam, amikacin or ciprofloxacin and vancomycin, were proposed for $48.5 \%$ of patients.

Categorisation of patients with HAP was proposed by the ATS to approach HAP microbial epidemiology and to guide initial antimicrobial treatment. In this series, all HAP episodes were considered to be severe since they occurred during ICU stay or were the indication of ICU admission. Consequently, no patient was categorised in group 2. Ninety-five per cent of 
Table 5.-Potential adequacy of selected antimicrobial regimens in 124 episodes of hospital-acquired pneumonia classified according to the duration of mechanical ventilation and prior antimicrobial therapy

\begin{tabular}{|c|c|c|c|c|}
\hline & Group A & Group B & Group C & Group D \\
\hline Episodes of HAP $n$ & 31 & 33 & 3 & 57 \\
\hline \multicolumn{5}{|l|}{ Single antimicrobial agent } \\
\hline Amoxycillin/clavulanic acid & 74.2 & 15.2 & 33.3 & 10.5 \\
\hline Cefotaxime & 83.9 & 33.3 & 33.3 & 26.3 \\
\hline Piperacillin & 25.8 & 15.2 & 0 & 8.8 \\
\hline Piperacillin/tazobactam & 77.4 & 30.3 & 33.3 & 29.8 \\
\hline Ceftazidime & 77.4 & 36.4 & 33.3 & 26.3 \\
\hline Cefepime & 83.9 & 42.4 & 33.3 & 29.8 \\
\hline Imipenem & 83.9 & 39.4 & 33.3 & 35.1 \\
\hline \multicolumn{5}{|l|}{ Combinations with amikacin } \\
\hline Amoxycillin/clavulanic acid & 80.7 & 33.3 & 33.3 & 29.8 \\
\hline Cefotaxime & 83.9 & 39.4 & 33.3 & 29.8 \\
\hline Piperacillin & 87.1 & 75.7 & 33.3 & 42.1 \\
\hline Piperacillin/tazobactam & 90.3 & 78.9 & 33.3 & 52.6 \\
\hline Ceftazidime & 90.3 & 78.9 & 66.7 & 50.9 \\
\hline Cefepime & 96.8 & 84.9 & 66.7 & 50.9 \\
\hline Imipenem & 93.6 & 78.9 & 33.3 & 52.6 \\
\hline \multicolumn{5}{|c|}{ Combinations with amikacin and vancomycin } \\
\hline Amoxycillin/clavulanic acid & 80.7 & 36.4 & 66.7 & 36.8 \\
\hline Cefotaxime & 83.9 & 42.4 & 66.7 & 36.8 \\
\hline Piperacillin & 87.1 & 78.8 & 66.7 & 50.9 \\
\hline Piperacillin/tazobactam & 90.3 & 81.8 & 66.7 & 61.4 \\
\hline Ceftazidime & 90.3 & 81.8 & 100 & 61.4 \\
\hline Cefepime & 96.8 & 87.9 & 100 & 59.7 \\
\hline Imipenem & 93.6 & 81.8 & 66.7 & 59.7 \\
\hline \multicolumn{5}{|l|}{ Combinations with ciprofloxacin } \\
\hline Amoxycillin/clavulanic acid & 80.7 & 33.3 & 33.3 & 21.1 \\
\hline Cefotaxime & 83.8 & 39.4 & 33.3 & 29.8 \\
\hline Piperacillin & 80.7 & 60.6 & 33.3 & 38.6 \\
\hline Piperacillin/tazobactam & 87.1 & 63.6 & 33.3 & 57.9 \\
\hline Ceftazidime & 83.9 & 63.6 & 33.3 & 52.6 \\
\hline Cefepime & 90.3 & 69.7 & 33.3 & 56.1 \\
\hline Imipenem & 87.1 & 75.8 & 33.3 & 54.4 \\
\hline \multicolumn{5}{|c|}{ Combinations with ciprofloxacin and vancomycin } \\
\hline Amoxycillin/clavulanic acid & 80.7 & 36.4 & 33.3 & 28.1 \\
\hline Cefotaxime & 83.8 & 42.4 & 33.3 & 36.8 \\
\hline Piperacillin & 80.7 & 63.6 & 33.3 & 49.1 \\
\hline Piperacillin/tazobactam & 87.1 & 66.7 & 33.3 & 68.4 \\
\hline Ceftazidime & 83.9 & 66.7 & 33.3 & 64.9 \\
\hline Cefepime & 90.3 & 72.7 & 66.7 & 66.7 \\
\hline Imipenem & 87.1 & 78.8 & 66.7 & 61.4 \\
\hline
\end{tabular}

Data are presented as $\%$.

patients were included in group 3 and $5 \%$ in group 1 . The late onset of HAP, with a delay from hospital admission of $15.4 \pm 12.2$ days, and the fact that the ATS guidelines [8] including all HAP was not specific to VAP explain the disparate number of patients included in each group. Conversely, with Trouillet's et al. [12] classification, distribution of patients was more homogeneous, with $25 \%$ in group A, $25 \%$ in B and $50 \%$ in D. However, it must be emphasised that this classification was devised for VAP. In the current study, 21 patients did not have VAP. They were included in groups $\mathrm{A}$ or $\mathrm{B}$ since the duration of $\mathrm{MV}$ before HAP was obviously $<7$ days, but this point could, perhaps, be considered as an incorrect use of this classification.

In most ICUs, clinical problems are associated with the emergence of pathogens with increased antibiotic resistance [17]. All methods able to predict that a causative pathogen is resistant could optimise initial therapy. In this series, among 154 causative pathogens, 75 were "potentially resistant". The use of broad-spectrum antimicrobial therapy is regularly incriminated as one of the factors increasing resistance [13]. In the current group, 90 patients had received antibiotics prior to HAP onset. In these patients, $61.5 \%$ of pathogens were "potentially resistant" whereas, in patients without prior antibiotics, $17.8 \%$ were "potentially resistant". If only "truly resistant" pathogens are considered, $36(23.4 \%)$ were incriminated. Their frequency was significantly higher in patients with prior antimicrobial treatment $(30.3 \%)$ than in patients without $(6.7 \%)$. However, prior antimicrobial therapy on its own was associated with a poor specificity in predicting infection due to resistant organisms since some resistant pathogens were incriminated in patients without prior antibiotic(s). Thus, other characteristics should be taken into consideration. The ATS guidelines [8] 
recommend stratification of patients according to the time of HAP onset, HAP severity and prior antimicrobial therapy. In this study group, $5 \%$ of patients were included in group 1 . No resistant causative pathogen was incriminated in this group. In patients included in group 3, the frequency of "truly resistant" pathogens was $23.4 \%$ with a significant difference between patients with and without prior antibiotics. Consequently in this series, this classification was able to identify a group where the likehood of a resistant causative organism was nil. In the series by TROUILLET et al. [12], it was demonstrated that no "potentially resistant" pathogen was incriminated in VAP episodes with a duration of $\mathrm{MV}<7$ days and without prior antibiotics. In such a group in this study, the frequency of "potentially resistant" pathogens was $14.7 \%$ and the rates of "truly resistant" pathogens in the four groups were, 4.9, 19.5, 25 and 35.6\%, respectively. These results show that, in this series, the classification by TrouILLeT et al. [12] exhibits a lower negative-predictive value than the ATS classification [8] in the detection of HAP episodes due to resistant pathogens. Some conflicting results between the study by Trouillet et al. [12] and the current study suggest additional comments. In the study by TROUILlET et al. [12], there were no precise data on antimicrobial susceptibility. Pathogens were classified as "potentially resistant" or not. Among all Enterobacteriaceae spp., classified as no "potentially resistant" pathogens, the presence of extended-spectrum $\beta$-lactamase producing strains was possible. Consequently, the absence of resistant pathogens in patients with duration of $\mathrm{MV}<7$ days and without prior antibiotic(s) was surprising. Moreover, with the same definition of "potentially resistant" pathogens, the frequency of such pathogens was different in the two studies. In the current study as compared to the study by TrouILlET et al. [12], any prior antibiotic(s) within 30 days before HAP instead of 15 days were considered. This shorter period, rather than decreasing would have increased the risk of isolation of "potentially resistant" pathogens in patients falsely considered as having no prior treatment. Consequently, this can not be considered as a possible explanation. The most satisfying explanation is that the duration of MV before the onset of pneumonia is not, perhaps, relevant in this series. As reported, the mean time of HAP onset from hospital admission was $15.4 \pm 12.2$ days. Consequently, some patients included in groups A or B, because pneumonia occurred before the seventh day of MV, developed pneumonia later than the seventh day of hospitalisation. This could explain the isolation of resistant pathogens in patients in the current series without prior antibiotics and with a short duration of MV. In summary, it seems better to evaluate HAP onset from hospital admission, as in ATS guidelines [8], rather than from the start of MV, as in the study by TrouiLLeT et al. [12].

The ATS guidelines recommend various antimicrobial regimens [8]. Most of ATS recommended regimens fit perfectly for the patients in this study. Monotherapy could be proposed in patients from group 1. In group 3, a broad-spectrum betalactam combined with amikacin or ciprofloxacin could be proposed for all patients, with the addition of vancomycin in patients with prior antibiotic(s). However, this latter point could be the major drawback of the ATS guidelines. In the current study, most of the patients were included in this group and, consequently, vancomycin should be used for all these patients. As the widespread use of vancomycin has led to the appearance of resistance in Gram-positive cocci [18], such recommendations could have a negative impact on microbial ecology. Physicians are therefore faced with a dilemma, wide spectrum antimicrobial treatment including vancomycin is required to avoid an increased mortality, but such treatment could lead to an undesirable vancomycin selection pressure. The only way to answer this challenge is de-escalation therapy with initial use of broad-spectrum agents, wait for cultures and, finally, focus on narrowspectrum agents if possible [19]. The low adequacy level obtained by all regimens in patients in group 3 with prior antibiotic(s) must also be emphasised. The "best" combination was cefepime plus amikacin plus vancomycin, with a level of $70 \%$. Such data emphasise the reality of emergence of resistant pathogens in the current author's unit and explains, perhaps, the high mortality rate associated with such HAP. With TROUILleT's et al. [12] classification, a broad-spectrum betalactam combined with amikacin or ciprofloxacin, could be proposed in groups A and B. The addition of vancomycin appears useless in these groups. For the remaining patients, the same agents combined with vancomycin could be proposed. The major drawback of this classification is the absence of monotherapies recommended. Its interest is to limit the recommendation for vancomycin to $\sim 50 \%$ of patients. The emergence of vancomycinresistant strains [20], potentially favoured by excessive use of a glycopeptide, underlines the interest of such a classification.

Numerous limitations of this study must be addressed. First, the analysis of the potential adequacy level of various regimens was retrospective. Second, only antibiotics used in the current authors' hospital and not all agents proposed by the ATS have been tested. Third, the results may be relevant to the current authors unit. Numerous studies have, indeed, demonstrated that HAP-causative organisms vary widely from one site to another [21]. Fourth, the classification by TrouILLET et al. [12] was modified to include nonventilated patients and to take into account prior antibiotic(s) within 1 month before HAP onset. Fifth, to assess the aetiological diagnosis, endotracheal aspiration with quantitative culture as a sampling method could be used. As recent recommendations [22] underline that quantitative procedures based on nonbronchoscopic or bronchoscopic techniques have similar sensitivities, specificities and positive-predictive values, this point could not be considered as being a limitation. Sixth, the definition of antimicrobial adequacy used in this study was different from the definitions used by KolleF and WARD [10], Luna et al. [11] or TROuILlet et al. [12]. However, to the best of the current authors' knowledge there is no clear definition of adequacy. Finally, it is not known how long these results will be 
valuable for the current therapeutic approach. Therefore, microbiological ecology must be regularly studied to provide up-to-date information.

To conclude, the American Thoracic Society classification [8] appears more specific than the classification by TROUILLET et al. [12] in predicting the absence of resistant causative pathogens in hospital-acquired pneumonia. Retrospectively, all antibiotic schemes recommended by the American Thoracic Society appear adequate in the current authors unit, but could lead to a frequent use of vancomycin. A stratification based on both classifications could be proposed. In patients with early-onset hospital-acquired pneumonia without specific risk factors (American Thoracic Society group 1), monotherapies could be used. In patients with either late onset hospital-acquired pneumonia or specific risk factors (American Thoracic Society group 3), prior antibiotic(s) and duration of mechanical ventilation could be taken into account. In the absence of prior antibiotic(s), a broad-spectrum betalactam combined with aminoglycoside or ciprofloxacin could be proposed. In patients with prior antibiotic(s), a similar regimen could be proposed when duration of mechanical ventilation is $<7$ days (Trouillet's group B). Vancomycin could be added in the remaining patients (Trouillet's group D). Such a classification would have allowed monotherapy for $5 \%$ of the patients in this study and limited vancomycin use for $48.5 \%$. An antibiotic strategy including initial antimicrobial treatment guided by such a stratification and, if possible, a de-escalation when antimicrobial data are available could increase the initial administration of adequate antimicrobial treatment and prevent the emergence of antibiotic resistance. Of course, a prospective validation of such a stratification is required.

\section{References}

1. Baker AM, Meredith JW, Haponik EF. Pneumonia in intubated trauma patients. Am J Respir Crit Care Med 1996; 153: 343-349.

2. Cunnion KM, Weber DJ, Broadhead WE, Hanson LC, Pieper CF, Rutala WA. Risk factors for nosocomial pneumonia: Comparing adult criticalcare population. Am J Respir Crit Care Med 1996; 153: $158-162$.

3. Fagon JY, Chastre J, Hance AJ, Montravers P, Novara A, Gibert C. Nosocomial pneumonia in ventilated patients: a cohort study evaluating attributable mortality and hospital stay. Am J Med 1993; 94 : 281-288.

4. Heyland DK, Cook DJ, Griffith L, Keenan SP, Brun Buisson C. The attributable morbidity and mortality of ventilator-associated pneumonia in the critically ill patient. The Canadian Critical Trials Group. Am J Respir Crit Care Med 1999; 159: 1249-1256.

5. Kollef MH, Silver P, Murphy DM, Trovillion E. The effect of late-onset ventilator-associated pneumonia in determining patient mortality. Chest 1995; 108: 16551662.

6. Leroy $\mathrm{O}$, Guilley $\mathrm{J}$, Georges $\mathrm{H}$, et al. Effect of hospital-acquired ventilator-associated pneumonia on mortality of severe community-acquired pneumonia. J Crit Care 1999; 14: 12-19.

7. Papazian L, Bregeon F, Thirion X, et al. Effect of ventilator-associated pneumonia on mortality and mortality. Am J Respir Crit Care Med 1996; 154: 9197.

8. American Thoracic Society. Hospital-acquired pneumonia in adults: diagnosis, assessment of severity, initial antimicrobial therapy, and preventative strategies. Am J Respir Crit Care Med 1995; 153: 17111725.

9. Bryan CS, Reynolds KL. Bacteriemic nosocomial pneumonia. Analysis of 172 episodes from a single metropolitan area. Am Rev Respir Dis 1984; 129: 668671.

10. Kollef $\mathrm{MH}$, Ward S. The influence of mini-BAL cultures on patient outcomes: implications for the antibiotic management of ventilator-associated pneumonia. Chest 1998; 113: 412-420.

11. Luna CM, Vujacich P, Niederman MS, et al. Impact of BAL data on the therapy and outcome of ventilator-associated pneumonia. Chest 1997; 111: 676-685.

12. Trouillet JL, Chastre J, Vuagnat A, et al. Ventilatorassociated pneumonia caused by potentially drugresistant bacteria. Am J Respir Crit Care Med 1998; 157: 531-539.

13. Shlaes DM, Gerding DN, John JF Jr, et al. Society of healthcare epidemiology of America and infectious diseases society of America joint committee on the prevention of antimicrobial resistance: Guidelines for the prevention of antimicrobial resistance in hospitals. Clin Infect Dis 1997; 25: 584-599.

14. LeGall JR, Lemeshow S, Saulnier F. A new simplified acute physiologic score (SAPS II) based on European/ North American multicenter study. JAMA 1993; 27: 2957-2963.

15. Antibiogram Committee of the French Society for Microbiology Official Statement. Pathol Biol 1999; 47 : 845-872.

16. Rello J, Gallego M, Mariscal D, Sonora R, Valles J. The value of routine microbial investigation in ventilator-associated pneumonia. Am J Respir Crit Care Med 1997; 156: 196-200.

17. Kollef MH, Fraser VJ. Antibiotic resistance in the Intensive Care Unit. Ann Intern Med 2001; 134: 298314.

18. Mayhall CG. Prevention and control of vancomycin resistance in Gram-positive coccal microorganisms: Fire prevention and fire fighting. Infect Control Hosp Epidemiol 1996; 17: 353-355.

19. Kollef MH. Hospital-acquired pneumonia and de-escalation of antimicrobial treatment. Crit Care Med 2001; 29: 1473-1474.

20. Smith TL, Pearson ML, Wilcox KR, et al. Emergence of vancomycin resistance in Staphylococcus aureus. N Engl J Med 1999; 340: 493-501.

21. Rello J, Sa-Borges M, Correa H, Leal S-R, Baraibar J. Variations in etiology of ventilator-associated pneumonia across four treatment sites Implications for antimicrobial prescribing practices. Am J Respir Crit Care Med 1999; 160: 608-613.

22. Grossman RF, Fein A. Evidence-based assessment of diagnostic tests for ventilator-associated pneumonia. Chest 2000; 117: 177S-181S. 